\title{
BMJ Open Pharmaceutical management of elderly high-risk patients in perioperative settings (PHAROS): protocol of a pilot sequential intervention study
}

\author{
Julia Richter, ${ }^{1}$ Moritz Sebastian Schönfeld (D) ,2 Claudia Langebrake, ${ }^{1,3}$ \\ Corinna Bergelt, ${ }^{2}$ Levente Kriston, ${ }^{2}$ Cynthia Olotu (1D , ${ }^{4}$ Rainer Kiefmann ${ }^{4}$
}

To cite: Richter J,

Schönfeld MS, Langebrake C, et al. Pharmaceutical management of elderly highrisk patients in perioperative settings (PHAROS): protocol of a pilot sequential intervention study. BMJ Open 2020;10:e039094. doi:10.1136/ bmjopen-2020-039094

- Prepublication history and additional material for this paper are available online. To view these files, please visit the journal online (http://dx.doi org/10.1136/bmjopen-2020039094).

JR and MSS contributed equally.

$\mathrm{CO}$ and RK share last authorship.

Received 06 April 2020 Revised 08 August 2020 Accepted 11 October 2020

Check for updates

(C) Author(s) (or their employer(s)) 2020. Re-use permitted under CC BY-NC. No commercial re-use. See rights and permissions. Published by BMJ.

For numbered affiliations see end of article.

Correspondence to Mr Moritz Sebastian Schönfeld; mo.schoenfeld@uke.de

\section{ABSTRACT}

Introduction With increasing age, the risk of complications after surgery rises in elderly patients. Furthermore, the prevalence of multimorbidity and polypharmacy rises with age, making this elderly population especially vulnerable for drug-related problems and posing an additional risk for postoperative complications. Still, only few studies have concentrated on investigating how medication safety can be improved in these patients. The aim of this pilot study is to examine the impact of a comprehensive intervention (interprofessional systematic medication therapy management) on medication appropriateness in elderly polymedicated, multimorbid patients during hospital stay for elective surgery.

Methods and analysis This pilot study will include a total number of 140 patients. Surgical high-risk patients $\geq 65$ years taking more than five chronic systemic drugs will be recruited consecutively for 9 months in the control group capturing usual care regarding medication history and in-hospital medication therapy management without any study intervention. Recruitment of the intervention group will be conducted for another 9 months. The intervention consists of the following components: an additional medication history by a hospital pharmacist before admission, a subsequent medication review, optimisation of the long-term medication and recommendations to the patient's general practitioner. A follow-up will be performed 3 months after surgery. As the primary study outcome, medication appropriateness will be measured using the Medication Appropriateness Index.

Secondary outcomes are postoperative complications, incidence and frequency of adverse drug reactions and potentially inappropriate medication in the elderly, satisfaction with inpatient and outpatient care, medication reconciliation and health-related quality of life. Multivariable analyses will be used to analyse all quantitative research questions.

Ethics and dissemination Ethics approval was obtained by the medical ethics committee of the Medical Chamber of Hamburg (study ID: PV5754). Data will be published in peer-reviewed journals and presented at conferences.

Trial registration number The study is registered at www.drks.de: DRKS00014621.

\section{Strengths and limitations of this study}

- First trial to investigate the effect of perioperative medication therapy management in elderly surgical high-risk inpatients in Germany.

- Data collection 3 months after hospital stay allows for measurement of medium-term effects.

- Due to the complexity of the intervention it may be difficult to study which component of the intervention will be responsible for the effects on the measured outcomes.

- Randomised controlled trial considered unsuitable due to contamination bias as the intervention will impact care processes.

\section{INTRODUCTION}

Multimorbidity and polypharmacy as well as changes in pharmacokinetics and pharmacodynamics increase elderly patients' vulnerability for medication errors. ${ }^{12}$ In addition, surgery also increases the risk of complications in elderly people. Thus, these patients demand for special attention in the perioperative process regarding their long-term medication. Especially, drug related problems (DRP) and potentially inappropriate medications (PIM) need to be avoided. DRP are circumstances or events associated with pharmacotherapy that actually or potentially prevent from achieving desired therapeutic goals. ${ }^{3}$ PIM for elderly patients are drugs that are associated with high risk of adverse drug reactions. ${ }^{45}$ Several classification systems have been developed to identify PIM. ${ }^{6-9}$ Polymedication and especially the intake of centrally acting drugs may be involved in the development of postoperative complications such as delirium or postoperative neurocognitive disorders. ${ }^{10} 11$

Polypharmacy in elderly people is one of the greatest financial risks in the healthcare system. ${ }^{12}$ Due to demographic development, 
this problem affects a constantly rising number of patients. ${ }^{13}$ Therefore, it is highly important to identify effective interventions that can optimise drug therapy and reduce adverse drug reactions. Comprehensive medication reviews (CMR), structured reviews of the patient's long-term medication, in consideration of the patient's individual conditions, may be suitable for achieving these goals. ${ }^{14}$ According to a Cochrane review from 2016, there is still insufficient evidence to substantiate the impact of CMR on hard patient-related outcomes, so further studies are needed. ${ }^{15}$

Furthermore, the transition between outpatient and inpatient care challenges patients, physicians and all other involved healthcare professionals. The differentiated capture of all regularly taken drugs represents a complex task within the preoperative evaluation of elderly patients. This is complicated by the fact that not every patient has an overview of the drugs prescribed by different specialists and/or his/her over the counter (OTC) medication. This commonly results in discrepancies between long-term medication and inpatient prescriptions. In more than $50 \%$ of patients, at least one medication error occurs already at hospital admission. ${ }^{16}$ Pharmacy-led medication reconciliation, defined by the Institute of Healthcare Improvement as "a process of identifying the most accurate list of all medications a patient is taking", ${ }^{17}$ at either admission or discharge was found to be an effective approach to reduce medication discrepancies. ${ }^{18}$

In this field of research, one of the few published studies with data from Germany was able to show a positive impact of interdisciplinary collaboration on medication safety for elderly multimorbid patients in outpatient care. ${ }^{19}$ Central element of this study was a medication therapy management (MTM).$^{20}$ In collaboration with general practitioners, nursing staff and pharmacists, Köberlein-Neu et al found a statistically significant impact of the MTM on the quality of drug therapy as measured by the Medication Appropriateness Index (MAI). ${ }^{21}{ }^{22}$ We now aim to transfer this approach into the inpatient sector.

The preoperative evaluation of all patients undergoing surgical intervention offers the anaesthesiologist the opportunity to introduce patients of all surgical disciplines to a hospital pharmacist. This way, an interdisciplinary team of pharmacist, physician and general practitioner can have a lasting positive impact on the long-term medication and thus on the perioperative process.

The aim of the PHAROS study is to determine the impact of the intervention (comprehensive interprofessional MTM) on the medication appropriateness measured using the MAI. Furthermore, the study will examine if the intervention will affect the secondary outcomes postoperative complications, incidence and frequency of DRP and PIM, satisfaction with inpatient and outpatient care, medication reconciliation and health-related quality of life. These outcomes are expected to be positively affected by optimising the long-term medication and avoiding medication errors at transition of care. Accordingly, we expect a reduction of postoperative complications and an improvement in quality of patient care.

To assess the acceptance of the intervention, short interviews will be conducted with a small sample of clinicians and pharmacists who treated patients involved in this study. Based on the results of these interviews, a short questionnaire will be developed which will be used to examine acceptance of the intervention of all involved hospital physicians and pharmacists as well as feasibility of the intervention.

\section{METHODS AND ANALYSIS Design}

A pilot sequential intervention study will be conducted with data first collected in the control group and subsequently in the intervention group. As the intervention will impact care processes, a randomised parallel-group trial with concurrent investigation of both groups was considered unsuitable due to contamination bias. The intervention is, for example, expected to change documentation and feedback processes which in turn could bias patient care in the comparison group. Accordingly and as recommended by the Medical Research Council for the context of complex interventions, no patient level randomisation is conducted. ${ }^{23}$ In addition, patient allocation will be determined by trial phase and not concealed in any form as the trial has a non-randomised sequential design. Neither patients nor recruiters, outcome assessors or data analysts will be blinded to treatment assignment.

\section{Measurement time points}

- T1: Baseline assessment (21 days or more before elective surgery).

- T2: Medication review (1 to 7 days after T1, for intervention group only).

- T3: Preoperative assessment (day of admission).

- T4: Postoperative assessment (day of hospital discharge).

- T5: Follow-up assessment (3 months after T4).

\section{Cooperation partners}

The trial will be conducted in cooperation with three departments at the University Medical Center HamburgEppendorf (UKE): The Department of Anesthesiology, the Hospital Pharmacy and the Department of Medical Psychology.

\section{Inclusion and exclusion criteria}

The study includes polymedicated, multimorbid patients aged 65 years and older scheduled for elective surgery.

\section{Inclusion criteria}

- Patient age $\geq 65$ years.

- Preoperative Score to Predict Postoperative Mortality ${ }^{24}$ (POSPOM, a risk score based on medical patient data (age, diagnosis, surgery) to predict in-hospital mortality)) $\geq 25$. 


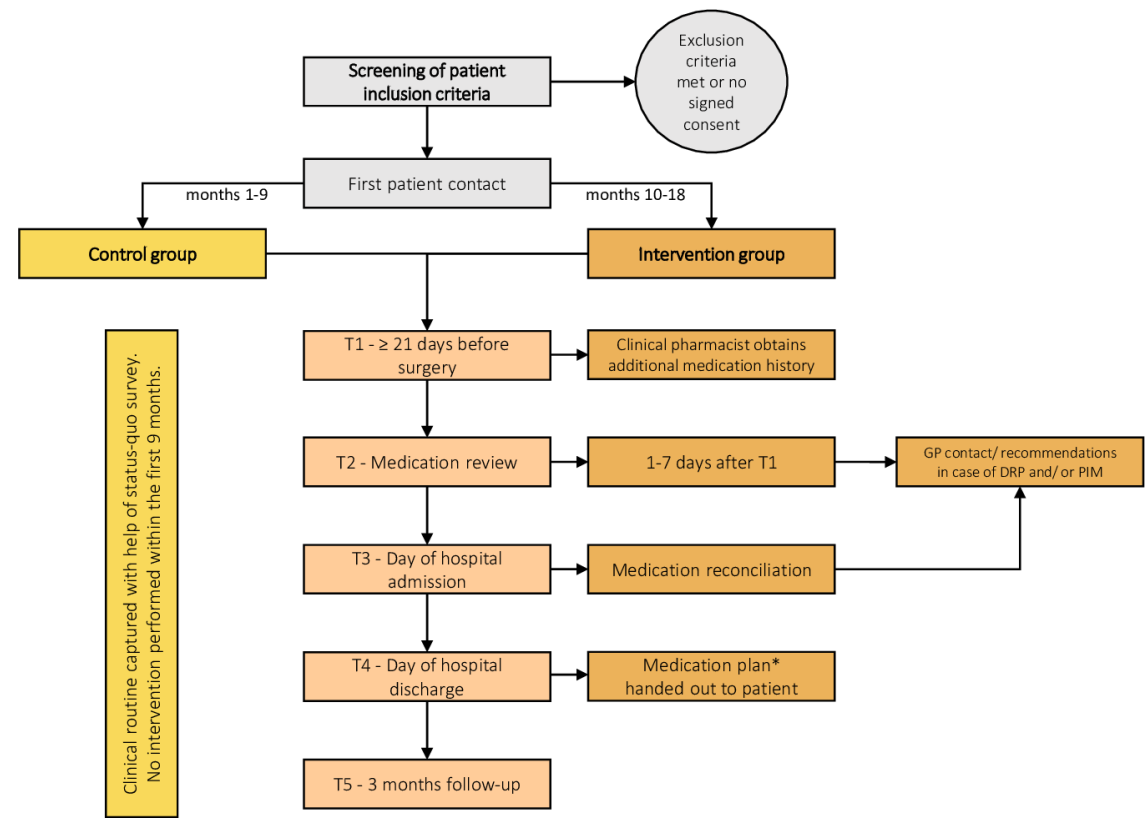

Figure 1 Recruitment process of control and intervention group. Notes: *German Nationwide Medication Plan. DRP, drugrelated problem; GP, general practitioner; PIM, potentially inappropriate medication.

- $\geq 5$ chronic systemic medicinal products/drugs.

- $\geq 21$ days before elective surgery.

\section{Exclusion criteria}

- Refusal of informed consent.

- Emergency surgery.

- Surgery with planned postoperative stay on intensive care unit.

- Cerebral and ophthalmological surgery.

- Surgery with postoperative care $\leq 24$ hours.

- Current or history of intravenous drug abuse.

- Psychosis.

- Chronic benzodiazepine abuse.

- Parkinson's disease.

- Illiteracy.

- Deafness, blindness.

- Insufficient language skills.

- Preexisting intellectual disability.

\section{Recruitment and data collection}

The recruitment process is depicted in figure 1. Eligible patients will be contacted and informed about the study in the outpatient department of anesthesiology during preoperative evaluation by a study nurse. In case of agreement, written informed consent (online supplemental file 1) will be obtained and $\mathrm{T} 1$ data collection will be conducted including patient self-reports and medical patient data (eg, medical history, POSPOM, type of surgery) and testing of cognitive functions will be performed. Within the first 9 months of the study patients will be recruited for the control group. Following a short transitional period and implementation of the intervention, patient recruitment for the intervention group will be performed for another 9 months.
Apart from first patient contact during preoperative evaluation (T1), patients will be seen on the day of hospital discharge (T4) and 3 months after hospital discharge (T5). Secondary outcomes and additional measurements will be captured with paper-based questionnaires at T1, $\mathrm{T} 4$ and T5. We aim to include all eligible patients. Also, we continuously record reasons of non-participation as well as unintended effects during recruitment and intervention. In case of unintended effects resulting from the intervention, termination of the clinical trial will be considered.

Patients' long-term medication will be gathered using a medication history form at $\mathrm{T} 1$ and $\mathrm{T} 5$. The pharmacy-led medication review will be conducted retrospectively for the control group and 1 to 7 days after T1 for the intervention group (T2). After hospital admission and prior to surgery (T3), a hospital pharmacist will gather the information about the patient's medication at point of admission from the hospital's prescription software. The discharge medication (T4) will be taken from the patients' discharge report.

Qualitative data will be collected following the recruitment of the intervention group. Accordingly, a selection of physicians and hospital pharmacists, who treated patients in this context, will be asked to participate in short interviews to assess their acceptance of the intervention. The interviews will address the physicians' and pharmacists' experiences with the implementation of the intervention (eg, context factors, events, general conditions) in order to assess the implementation process. Based on the results of these interviews, a short questionnaire will be created to further address the acceptance and feasibility of the intervention from the clinicians' point of view. This questionnaire will be distributed to 
all physicians and pharmacists who were involved in the patient care of the intervention group.

Microsoft Access and IBM SPSS are used for data entry and data analyses, respectively. All patient data will be recorded pseudonymised and stored on secured devices.

This study protocol was written in accordance to the SPIRIT (Standard Protocol Items: Recommendations for Interventional Trials) guidelines. ${ }^{25}$

\section{Interventions and procedures \\ Control group}

In the first phase of this trial a control group will be recruited receiving standard of care and a status quo state will be obtained. Standard of care at the UKE means an electronic patient record including a computerised physician order entry with clinical decision support systems (CPOE-CDSS). At admission (T3), the attending ward physician prescribes the current medication. During the daily routine, hospital pharmacists perform a plausibility assessment of the inpatients' prescription regarding guideline-based therapy, possible drug interactions and dose adjustment to organ function supported by a CPOE-CDSS. After the ward pharmacist's approval and, if necessary, consultation with the ward physician and/or adjustments, the medication is delivered contemporarily by the hospital pharmacy.

The medication review of the control group will be performed based on the patient's medication plan and clinical patient data.

\section{Intervention group}

Patients in the intervention group also receive usual care as described for the control group. For patients in the intervention group, medication history will be obtained by a hospital pharmacist during the preoperative evaluation (T1). The pharmacist will enter the medication into the CPOE-CDSS for the ward physician to check, verify and, if necessary, add new prescriptions (analgesics, antibiotics, and so on). In contrast to the control group, a medication review will be conducted 1 to 7 days after T1 using the patient's medication plan, clinical patient data and additional from the patient interview. In case of DRP and/or PIM the patient's general practitioner (GP) will be contacted to give him/her recommendations about medication optimisation. The GP decides whether to implement the recommendations. At discharge (T5), the German Nationwide Medication Plan $^{26}$ (NMP) will be attached to the discharge letter in order to avoid information loss. The NMP was developed by the Federal Association for Statutory Health Insurance Physicians (KBV), the German Medical Association and the German Association of Pharmacists as part of the e-health legislation of December 2015. It serves as a uniformly standardised medication plan for patients who take more than three drugs permanently. The medication will be reevaluated by the hospital pharmacist 3 months after discharge (T5) in order to measure the level of acceptance and to determine the extent to which the given recommendations have been implemented. A 3-month follow-up period was considered appropriate to avoid other modifications to the long-term medication resulting from longer follow-up periods and possibly influencing the results of the study.

\section{Outcomes and measurements}

Outcome measures are displayed in table 1.

\section{Primary outcome}

Medication Appropriateness Index

The primary outcome of this study is the patient's medication appropriateness measured by the MAI. The MAI assesses the appropriateness of medication prescriptions (judgement-based) and has good inter-rater and intra-rater reliability. ${ }^{21}$ Specifically, the general medicine guideline on multimedication (German Society of General Medicine, ${ }^{27}$ DEGAM) recommends the MAI to be used for critical medication analysis in elderly patients with polypharmacy. Here, the MAI will be used to determine whether the study intervention can improve the quality of drug therapy. The rater assesses whether the medication is appropriate, marginally appropriate or inappropriate in 10 categories and assigns the following scores accordingly: (0) for 'appropriate' and 'marginally appropriate' and (1) to (3) for 'inappropriate' depending on the category. ${ }^{21} 22$ A main score is then calculated which reflects the appropriateness of the patient's medication. To standardise the instrument the MAI provides operational definitions and examples for each criterion.

A hospital pharmacist will determine the MAI of the patient's long-term medication. In order to improve reliability of the measures, a second independent hospital pharmacist will conduct analyses of the MAI from randomly chosen study patients of both groups.

\section{Secondary outcomes}

Postoperative complications

Postoperative complications are defined based on detailed standards described by Jammer et al (2015).$^{28}$ Accordingly, a list of 34 different complications was created to rate postoperative complications as 'mild', 'moderate' or 'severe'.

\section{Incidence and frequency of DRP and PIM}

DRP are typically identified using the MAI. Five additional criteria of DokuPIK ${ }^{29}$ (German database to document clinical pharmacists' interventions in hospitals) are added to examine certain aspects in more detail: 'Failure to adjust dose for organ function', '(inappropriate) administration interval', 'failure to discontinue relevant drugs preoperatively/perioperatively', 'drug allergy or medical history not considered' and '(clear) indication not (or no longer) given'. PIM are assessed by FORTA ${ }^{8}$ (Fit for the Aged) and the EU(7)-PIM ${ }^{9}$ list. In the 2018 updated FORTA list 296 drugs for 30 essential diagnoses in elderly patients are evaluated and classified into four groups: $(\mathrm{A})=$ 'essential', $(\mathrm{B})=$ 'beneficial', $(\mathrm{C})=$ 'questionable' and (D) = 'avoid'. The EU(7)-PIM list rates a total amount of 282 drugs as potentially inappropriate for 
Table 1 Study measures in control and intervention group

\begin{tabular}{|c|c|c|c|c|c|c|}
\hline Measures & Instruments & $\begin{array}{l}\text { T1 - } \\
\text { baseline }\end{array}$ & $\begin{array}{l}\text { T2 - medication } \\
\text { review }\end{array}$ & $\begin{array}{l}\text { T3 - } \\
\text { day of hospital } \\
\text { admission }\end{array}$ & $\begin{array}{l}\text { T4 - } \\
\text { day of hospital } \\
\text { discharge }\end{array}$ & $\begin{array}{l}\text { T5 - } \\
3 \text { months } \\
\text { follow-up }\end{array}$ \\
\hline \multicolumn{7}{|l|}{ Primary outcome } \\
\hline \multicolumn{7}{|l|}{ Secondary outcomes } \\
\hline Postoperative complications & $\mathrm{EPCO}^{28}$ & & & & $\mathbf{x}$ & $\mathbf{x}$ \\
\hline $\begin{array}{l}\text { Potentially inappropriate } \\
\text { medication }\end{array}$ & $\mathrm{PIM}^{9}$ & $\mathbf{x}$ & $\mathbf{x}$ & $\mathbf{x}$ & $\mathbf{x}$ & $\mathbf{x}$ \\
\hline Satisfaction with outpatient care & ZAPA $^{30}$ & $x$ & & & & $x$ \\
\hline Satisfaction with inpatient care & ZUF-8 $8^{31}$ & & & & $\mathbf{x}$ & \\
\hline Medication reconciliation & SOP MedRec ${ }^{33}$ & & & $\mathbf{x}$ & & \\
\hline Treatment adherence & MARS-D ${ }^{38}$ & $x$ & & & $\mathbf{x}$ & $x$ \\
\hline Depression & PHQ-4 ${ }^{39}$ & $\mathbf{x}$ & & & $\mathbf{x}$ & $\mathbf{x}$ \\
\hline Anxiety & PHQ $-4^{39}$ & $x$ & & & $\mathbf{x}$ & $x$ \\
\hline Frailty & LUCAS-FI ${ }^{43}$ & $\mathbf{x}$ & & & & $\mathbf{x}$ \\
\hline \multirow[t]{2}{*}{ Functional status } & $\mathrm{IADL}^{46}$ & $\mathbf{x}$ & & & & $\mathbf{x}$ \\
\hline & $\mathrm{BADL}^{47}$ & $x$ & & & $\mathbf{x}$ & $\mathbf{x}$ \\
\hline \multirow[t]{3}{*}{ Cognitive ability } & DemTect $^{51}$ & $\mathbf{x}$ & & & $\mathbf{x}$ & $\mathbf{x}$ \\
\hline & TMT $A$ and $B^{4950}$ & $\mathbf{x}$ & & & $\mathbf{x}$ & $\mathbf{x}$ \\
\hline & WHODAS $2.0^{48}$ & $\mathbf{x}$ & & & $\mathbf{x}$ & $x$ \\
\hline \multirow{3}{*}{ Medical patient data } & $\mathrm{POSPOM}^{24}$ & $x$ & & & & \\
\hline & $\begin{array}{l}\text { Premedication, } \\
\text { mode and } \\
\text { duration of } \\
\text { anaesthesia, type } \\
\text { of surgery, early } \\
\text { intraoperative and } \\
\text { postoperative } \\
\text { complications, } \\
\text { pain }\end{array}$ & & & & $\mathbf{x}$ & \\
\hline & $\begin{array}{l}\text { Duration of } \\
\text { hospital stay, } \\
\text { complications, } \\
\text { newly emerged } \\
\text { medical conditions }\end{array}$ & & & & & $x$ \\
\hline
\end{tabular}

BADL, Basic Activities of Daily Living; DokuPIK, Documentation of Pharmacists' Interventions in Hospital; EPCO, European Perioperative Clinical Outcome definitions; IADL, Instrumental Activities of Daily Living; LUCAS-FI, Longitudinal Urban Cohort Ageing Study Functional Index; MAI, Medication Appropriateness Index; MARS-D, German version of the Medication Adherence Rating Scale; MEL, German Münchener Ereignisliste; PHQ-4, Patient Health Questionnaire; PIM, Potentially Inappropriate Medication; SF-12, Short Form 12; SIMS-D, German version of the Satisfaction with Information about Medicines Scale; SOP MedRec, Standard Operating Procedure Medication Reconciliation; TMT, Trail Making Test; WHODAS 2.0, World Health Organization, Disability Assessment Schedule 2.0; ZAPA, Questionnaire of Satisfaction with Ambulatory Care; ZUF-8, Patient Satisfaction Questionnaire. 
elderly patients. It was developed based on expert knowledge and provides suggestions for therapeutic alternatives and dose adjustments.

\section{Satisfaction with outpatient care}

The ZAPA is a short instrument to assess patient satisfaction with outpatient medical care. ${ }^{30}$ It contains four items which are rated on a 4-point scale ranging from (0) 'very dissatisfied/no confidence/very low' to (3) 'very satisfied/high confidence/very high'. A sum score, ranging from 0 to 12 can be calculated and linearly transformed into a total score ranging from 0 (highest level of patient dissatisfaction) to 100 (highest level of patient satisfaction).

\section{Satisfaction with inpatient care}

Patient satisfaction with inpatient care is measured with the ZUF- $8{ }^{31}$ The ZUF- 8 is the reliable and valid German version of the Client Satisfaction Questionnaire ${ }^{32}$ and comprises eight items. All items are rated on a 4-point scale and range from (1) 'bad' to (4) 'excellent'. A sum score is calculated ranging from 8 (lowest level of satisfaction) to 32 (highest level of satisfaction).

\section{Medication reconciliation}

Analysis of the correct medication at the interfaces admission and discharge will be carried out based on the standard operation procedure 'Medication Reconciliation' of the German Centre for Quality Assurance in Medicine (ÄZQ) ${ }^{33}$ The occurrence of medication discrepancies at hospital admission (T3) will be measured using the following four items (yes-no query): 'transcription error at admission (T3)', '(unintended) omission', '(unintended) addition' and 'incorrect dosage'.

\section{Health-related quality of life}

On the basis of 12 items, the SF-12 assesses two dimension of health-related quality of life: physical health (PCS) and mental health (MCS).$^{34}$ In particular, each dimension is represented by four health domains (PCS: 'Physical Functioning' (PF), 'Role-Physical' (RP), 'Bodily Pain' (BP), 'General Health' (GH); MCS: 'Vitality' (VT), 'Social Functioning' (SF), 'Role-Emotional' (RE), 'Mental Health' (MH)). Individual sum scores are calculated for the both dimension PCS and MCS from patient responses by using weighted means. ${ }^{35}$

\section{Additional measures}

\section{Satisfaction with medication}

In order to measure patient satisfaction with medication, the Satisfaction with Information about Medicine Scale (SIMS-D) is used in this study. ${ }^{36}$ The SIMS-D is the German version of the SIMS $^{37}$ and comprises 17 self-report items about satisfaction with medication therapy management and medication information. All items are rated on a 5-point-scale and answers are coded (0) for 'too much', 'too little' and 'none received' and (1) for 'about right' and 'none needed'. Accordingly, the total score for the SIMS-D range from 0 to 17 with higher scores indicating higher levels of satisfaction with medication. The SIMS-D has been shown to be a reliable and valid. ${ }^{36}$

\section{Treatment adherence}

Treatment adherence is assessed with the MARS-D, ${ }^{38}$ the reliable and valid German version of the Medication Adherence Report Scale (MARS) which measures patient behaviour with regard to medication adherence. The MARS-D is composed of five self-report items rated on a scale from (1) 'always' to (5) 'never'. Consequently, the sum score ranges from 5 to 25 with higher values indicating higher medication adherence.

\section{Depression and anxiety}

The Patient Health Questionnaire-4 ${ }^{39} 40$ (PHQ-4) is a very brief self-report screening tool for depression and anxiety. It combines four items, two obtained from the Generalised Anxiety Disorder-7 scale ${ }^{41}$ (GAD-7) and two obtained from the Patient Health Questionnaire- $8^{42}$ (PHQ-8). Total scores range from 0 to 12 and can be interpreted in terms of depression/anxiety severity as normal, mild, moderate and severe.

\section{Frailty}

The LUCAS-FI is a self-administered screener which assesses levels of frailty in seniors. ${ }^{43}$ The questionnaire was developed within the Longitudinal Urban Cohort Ageing Study ${ }^{44}$ (LUCAS) and comprises 12 items which represent frailty-risks (six items) and frailty-reserves (six items). As all items are dichotomised for further calculation, total scores range from 0 to 12 and can be classified in the context of frailty (FRAIL, pre-FRAIL) and fitness (FIT). Accordingly, respondents may be assigned to one of the following three categories: FRAIL ( 3 to 6 risks and less than 3 reserves), FIT ( 3 to 6 reserves and less than 3 risks), pre-FRAIL (either less than 3 risks and 3 reserves or more than 3 risk and 3 reserves). Reliability and validity for the LUCAS-FI have been confirmed. ${ }^{434}$

\section{Functional status I}

The IADL (Instrumental Activities of Daily Living) is used to assess instrumental activities related to independent living. ${ }^{45}$ Each component contains three to five short statements describing typical activities or tasks. As these statements represent the respective scales of the components, a number from (0) to a maximum of (5) is assigned to each statement. This scoring was adapted from the manual as we expect more detailed information about the patient's functional status that way. Accordingly, total scores range from 8 to 31 with lower scores indicating better capacity. The IADL has been confirmed to be a reliable and valid screening tool. ${ }^{45}$

\section{Functional status II}

The basic activities of daily living are assessed with the Barthel Index (BI). ${ }^{46} 47$ The BI comprises 10 typical daily activities. Each activity includes two to four statements with assigned numbers from (0) to a maximum of (10). Hence, total scores range from 0 (lowest level of capacity) 
to 100 (highest level of capacity). Satisfactory levels of inter-rater reliability and test-retest-reliability have been demonstrated for the BI.

\section{Cognitive ability I}

Self-reported cognitive ability is assessed by the cognition domain of the WHODAS 2.0. ${ }^{48}$ This domain comprises six items on understanding and communication and is rated on a 5-point scale from (1) 'no difficulty' to (5) 'extreme difficulty/cannot do'. Sum scores are calculated resulting in total scores that range from 6 to 30 with higher scores indicating higher difficulties in the domain of communication and thinking. The WHODAS 2.0 has been shown to be a reliable and valid instrument.

\section{Cognitive ability II}

In order to further assess attention deficits and executive dysfunctions the Trail Making Test (TMT) is conducted. ${ }^{49} 50$ The TMT contains two parts each consisting of 25 circles. While Part A comprises circles with numbers from 1 to 25 , part $\mathrm{B}$ includes circles with numbers (1 to 13 ) and letters (A to L). The task is to connect the circles as quickly as possible by drawing lines without lifting the pencil. Accordingly, in part A, lines are drawn in ascending order from 1 to 25 whereas in part B lines are drawn in ascending order alternating between numbers and letters. The time is measured in seconds until the test is completed. Higher scores indicate higher levels of impairment with scores above 78 and $273 \mathrm{~s}$ indicating cognitive deficiency for Part A and B, respectively.

\section{Cognitive ability III}

The DemTect is a neuropsychological tool that was initially developed to screen for symptoms of dementia but gains increasing popularity as an assessment of overall cognitive functions. ${ }^{51}$ It includes five short tasks with regard to different cognitive domains: 'verbal memory', 'verbal fluency', 'cognitive flexibility' and 'attention processes'. Total scores range from 0 to 18 , with scores above 13 indicating age-appropriate cognitive ability, scores from 9 to 12 indicating mild cognitive impairment and scores below 8 indicating suspected dementia. Reliability and validity of the DemTect have been confirmed. ${ }^{51}$

\section{Life events}

Self-developed items adapted from the German Münchener Ereignisliste (MEL) are used to descriptively assess life events of all patients. ${ }^{52}$ Patients are asked to state if and how many life events occurred within the last 6 months in the following areas: 'Family', 'Death', 'Work/household', 'Living situation', 'Financial aspects', 'Social life' and 'Health/sickness'.

\section{Data analyses}

\section{Quantitative analyses}

Descriptive and univariate analyses will be carried out in order to examine differences between the control and the intervention group at baseline. In case of substantial group differences, covariates (eg, age, number of drugs) will be included in the model or propensity score methods will be applied to reduce the probability of selection bias. ${ }^{53}$ Analysis of continuous outcomes, including the primary outcome, will be carried out by fitting a linear mixed model including time as a repeated-measures fixed factor, group as a fixed factor, their interaction term and a random intercept across subjects. The primary analyses will follow the intention-to-treat approach, including data from all participants. Categorical outcomes will be analysed in a similar manner using generalised linear mixed models. In addition, correlation analyses will be carried out to investigate associations between outcome measures. Also, we will perform further exploratory analyses. For example, we plan to analyse intraindividual differences in the physical function (according to the Barthel Index) of all included patients before and after surgery.

\section{Qualitative analyses}

All qualitative interviews will be recorded and transcribed verbatim. The data will then be analysed using thematic content analyses by inductively developing categories from the transcripts. From this, we will create a coding agenda with coding rules which will be used to analyse all interviews. Accordingly, text passages will be assigned to specific categories following the coding agenda. ${ }^{54}$

\section{Sample size}

A study sample size of 64 patients per group (control and intervention) is sufficient to detect moderate group differences (Cohen's $d=0.5$ ) with a power of 0.80 at a significance level of $p<0.05$. Since inclusion of covariates or the use of propensity score methods might be necessary, we aim to recruit 70 patients per group (140 in total).

For qualitative data collection, we anticipate to conduct a total of 40 interviews with clinical physicians and pharmacists involved in the care of patients who are recruited in the intervention group. Additionally, a short questionnaire will be created based on the interviews to examine another 250 physicians and pharmacist for their acceptance of the PHAROS intervention.

\section{Patient and public involvement}

No patients or public organisations were involved in the study preparation. Also, patients were not involved in the decision of the study design or the research questions. All participants will be recruited personally by hospital staff prior to their hospital stay. We will assess patient satisfaction with outpatient and inpatient care as well as satisfaction with medication. Also, we plan to collect information about drug-related problems through patient information. Hospital pharmacists and general practitioners are included in the process of the medication recommendation for the patients and individual recommendations for medication adaptations will be provided to the patients in written form.

\section{Ethics and dissemination}

The study protocol was reviewed and approved by the medical ethics committee of the Medical Chamber of 
Hamburg on 23 April 2018 (number: PV5754). The project is conducted in accordance with the Declaration of Helsinki. All patients and participating medical professionals are provided with written information about the study and questions are answered in face-to-face contact. Written informed consent must be given prior to study participation. The study intervention will at no time hamper with patient care or patient surgery.

The duration of the project is 36 months. Since recruitment of the control group started in October 2018, recruitment of the intervention group is expected to be completed in the summer of 2020. Accordingly, data entry and management as well as data analyses will be conducted successively, and results will be published in peer-reviewed journals.

\section{DISCUSSION}

To date only few studies have investigated the impact of an interprofessional MTM on the quality of medication therapy and postoperative outcomes in the elderly. To the best of our knowledge, this is the first study in Germany to examine this objective in elderly surgical high-risk patients in a perioperative setting by integrating multiple disciplines of care. The aim of this pilot study is to improve medication appropriateness of elderly patients through an interprofessional MTM intervention. Also, we aim to improve patient satisfaction with outpatient and inpatient care, treatment adherence and quality of life. As multiple outcomes are considered at multiple times, we expect a comprehensive picture of the long-term MTM of elderly surgical high-risk patients in the perioperative setting. This may contribute to further the understanding of problems in elderly patients' healthcare and to ease their transition between outpatient and inpatient care. Furthermore, the findings of this pilot study will build a solid basis for planning and conducting a large-scale multicentre randomised trial on the effects of an MTM in elderly patients undergoing surgery.

\author{
Author affiliations \\ ${ }^{1}$ Hospital Pharmacy, University Medical Center Hamburg-Eppendorf, Hamburg, \\ Germany \\ ${ }^{2}$ Department of Medical Psychology, University Medical Center Hamburg-Eppendorf, \\ Hamburg, Germany \\ ${ }^{3}$ Department of Stem Cell Transplantation, University Medical Center Hamburg- \\ Eppendorf, Hamburg, Germany \\ ${ }^{4}$ Department of Anaesthesiology, University Medical Center Hamburg-Eppendorf, \\ Hamburg, Germany
}

Correction notice Joint last authorship has been added for this article.

Acknowledgements We thank Professor Dr med Christian Zöllner, Professor Dr med Alwin E Goetz, Birgit Koch, Anna Mende, Dr rer nat Steffen Härterich and Jennifer Schröter for their support in the planning of the study. We also thank all surgical clinics for participating in the study.

Contributors $\mathrm{CO}$ and RK are the principal investigators of the study. The study concept was developed by C0, RK, CL, CB and LK. CO, JR, CL, MSS and CB developed the intervention and the study materials. LK, MSS and JR will analyse and interpret the data. The first draft of the study protocol was written by JR and MSS. CO, RK, CL, CB and LK critically revised it. All authors read and approved the final manuscript. JR and MSS contributed equally to this article.
Funding The study is funded after peer review by the German Federal Ministry of Health (Bundesministerium für Gesundheit, BMG, grant number G111F01001). The BMG is not involved in the study design, data collection, data analysis and interpretation and in writing this manuscript.

Competing interests None declared.

Patient consent for publication Not required.

Provenance and peer review Not commissioned; externally peer reviewed.

Supplemental material This content has been supplied by the author(s). It has not been vetted by BMJ Publishing Group Limited (BMJ) and may not have been peer-reviewed. Any opinions or recommendations discussed are solely those of the author(s) and are not endorsed by BMJ. BMJ disclaims all liability and responsibility arising from any reliance placed on the content. Where the content includes any translated material, BMJ does not warrant the accuracy and reliability of the translations (including but not limited to local regulations, clinical guidelines, terminology, drug names and drug dosages), and is not responsible for any error and/or omissions arising from translation and adaptation or otherwise.

Open access This is an open access article distributed in accordance with the Creative Commons Attribution Non Commercial (CC BY-NC 4.0) license, which permits others to distribute, remix, adapt, build upon this work non-commercially, and license their derivative works on different terms, provided the original work is properly cited, appropriate credit is given, any changes made indicated, and the use is non-commercial. See: http://creativecommons.org/licenses/by-nc/4.0/.

ORCID iDs

Moritz Sebastian Schönfeld http://orcid.org/0000-0002-8611-8717

Cynthia Olotu http://orcid.org/0000-0001-9272-7386

\section{REFERENCES}

1 Wickop B, Langebrake C. [Good prescribing practice in the elderly]. Ther Umsch 2014;71:366-73.

2 Mangoni AA, Jackson SHD. Age-Related changes in pharmacokinetics and pharmacodynamics: basic principles and practical applications. Br J Clin Pharmacol 2004;57:6-14.

3 Aly A-F. Definitionen zu Pharmakovigilanz und Arzneimitteltherapiesicherheit (AMTS). Arzneiverordnung in der Praxis 2015;42:99-104.

4 Wickop B, Härterich S, Sommer C, et al. Potentially inappropriate medication use in Multimorbid elderly inpatients: differences between the FORTA, PRISCUS and STOPP ratings. Drugs Real World Outcomes 2016;3:317-25.

5 Blanco-Reina E, Ariza-Zafra G, Ocaña-Riola R, et al. 2012 American geriatrics Society beers criteria: enhanced applicability for detecting potentially inappropriate medications in European older adults? A comparison with the screening tool of older person's potentially inappropriate prescriptions. J Am Geriatr Soc 2014;62:1217-23.

6 Holt S, Schmiedl S, Thürmann PA. Potentially inappropriate medications in the elderly: the PRISCUS list. Dtsch Arztebl Int 2010;107:543-51.

7 Gallagher P, O'Mahony D. STOPP (screening tool of older persons' potentially inappropriate prescriptions): application to acutely ill elderly patients and comparison with beers' criteria. Age Ageing 2008;37:673-9.

8 Pazan F, Weiss C, Wehling M, et al. The FORTA (fit fOR the aged) list 2015: update of a validated clinical tool fOR improved pharmacotherapy in the elderly. Drugs Aging 2016;33:447-9.

9 Renom-Guiteras A, Meyer G, Thürmann PA. The EU(7)-PIM list: a list of potentially inappropriate medications for older people consented by experts from seven European countries. Eur J Clin Pharmacol 2015;71:861-75.

10 Jyrkkä J, Enlund H, Lavikainen P, et al. Association of polypharmacy with nutritional status, functional ability and cognitive capacity over a three-year period in an elderly population. Pharmacoepidemiol Drug Saf 2011;20:514-22.

11 Evered L, Silbert B, Knopman DS, et al. Recommendations for the nomenclature of cognitive change associated with anaesthesia and surgery-2018. Br J Anaesth 2018;121:1005-12.

12 Chiatti C, Bustacchini S, Furneri G, et al. The economic burden of inappropriate drug prescribing, lack of adherence and compliance, adverse drug events in older people: a systematic review. Drug Saf 2012;35 Suppl 1:73-87.

13 Hovstadius B, Hovstadius K, Åstrand B, et al. Increasing polypharmacy - an individual-based study of the Swedish population 2005-2008. BMC Clin Pharmacol 2010;10:1-8. 
14 Blenkinsopp A, Bond C, Raynor DK. Medication reviews. Br J Clin Pharmacol 2012;74:573-80.

15 Christensen M, Lundh A. Medication review in hospitalised patients to reduce morbidity and mortality. Cochrane Database Syst Rev 2016;2:CD008986

16 Tam VC, Knowles SR, Cornish PL, et al. Frequency, type and clinical importance of medication history errors at admission to hospital: a systematic review. CMAJ 2005;173:510-5.

17 Resar R, Midlefort L. Medication reconciliation review. Boston, Massachusetts, USA Institute for healthcare improvement: medication reconciliation review, 2004.

18 Mekonnen AB, McLachlan AJ, Brien J-AE. Pharmacy-led medication reconciliation programmes at hospital transitions: a systematic review and meta-analysis. J Clin Pharm Ther 2016;41:128-44.

19 Köberlein-Neu J, Mennemann H, Hamacher S, et al. Interprofessionelles Medikationsmanagement bei multimorbiden Patienten. Eine Cluster-randomisierte studie (WestGem-Studie). Deutsches Ärzteblatt 2016;44:741-8.

20 Bundesvereinigung Deutscher Apothekerverbände. Grundsatzpapier zur Medikationsanalyse und zum Medikationsmanagement, 2014. Available: https://www.abda.de/fileadmin/user_upload/assets/Medi kationsmanagement/Grundsatzpapier_MA_MM_GBAM.pdf

21 Hanlon JT, Schmader KE, Samsa GP, et al. A method for assessing drug therapy appropriateness. J Clin Epidemiol 1992;45:1045-51.

22 Samsa GP, Hanlon JT, Schmader KE, et al. A summated score for the medication appropriateness index: development and assessment of Clinimetric properties including content validity. J Clin Epidemiol 1994;47:891-6.

23 Moore GF, Audrey S, Barker M, et al. Process evaluation of complex interventions: medical Research Council guidance. BMJ 2015;350:h1258.

24 Manach YL, Collins G, Rodseth R, et al. Preoperative Score to Predict Postoperative Mortality (POSPOM)Derivation and Validation. Anesthes 2016;124:570-9.

25 Chan A-W, Tetzlaff JM, Altman DG, et al. Spirit 2013 statement: defining standard protocol items for clinical trials. Ann Intern Med 2013;158:200-7.

26 Schwenzer S. Der Bundeseinheitliche Medikationsplan. Arzneiverordnung in der Praxis 2017;44:11-18.

27 Leitliniengruppe Hessen DEGAM. Hausärztliche Leitlinie Multimedikation, 2014. Available: www.pmvforschungsgruppe.de/ pdf/03 publikationen/multimedikation II.pdf

28 Jammer I, Wickboldt N, Sander M, et al. Standards for definitions and use of outcome measures for clinical effectiveness research in perioperative medicine: European perioperative clinical outcome (EPCO) definitions: a statement from the ESA-ESICM joint Taskforce on perioperative outcome measures. Eur J Anaesthesiol 2015;32:88-105.

29 Inbe-Heffinger A, Langebrake C, Hohmann C, et al. Prospective survey-based study on the categorization quality of hospital pharmacists' interventions using DokuPIK. Int J Clin Pharm 2019;41:414-23.

30 Scholl I, Hölzel L, Härter M, et al. Fragebogen Zur Zufriedenheit in Der ambulanten Versorgung - Schwerpunkt Patientenbeteiligung (ZapA). Klin Diagn Eval 2011;4:50-62.

31 Schmidt J, Lamprecht F, Wittmann WW. Zufriedenheit MIT Der stationären Versorgung. Entwicklung eines Fragebogens und erste Validitätsuntersuchungen. Psychother med Psychol 1989;39:248-55.

32 Larsen DL, Attkisson CC, Hargreaves WA, et al. Assessment of client/patient satisfaction: development of a general scale. Eval Program Plann 1979;2:197-207.

33 Ärztliches Zentrum für Qualität in der Medizin. SOP medication reconciliation, 2015. Available: https://www.aezq.de/ patientensicherheit/h5s/high5s-medrec
34 Morfeld M, Bullinger M, Nantke J, et al. [The version 2.0 of the SF-36 Health Survey: results of a population-representative study]. Soz Praventivmed 2005;50:292-300.

35 Ware J, Kosinski M, Keller SD. Sf-36 physical and mental health summary scales: A user's manual. Boston, MA: The Health Institute, 1994.

36 Mahler C, Jank S, Hermann K, et al. Psychometric properties of a German version of the "Satisfaction with Information about Medicines Scale" (SIMS-D). Value Health 2009;12:1176-9.

37 Horne R, Hankins M, Jenkins R. The satisfaction with information about medicines scale (SIMs): a new measurement tool for audit and research. Qual Health Care 2001;10:135-40.

38 Mahler C, Hermann K, Horne R, et al. Assessing reported adherence to pharmacological treatment recommendations. translation and evaluation of the medication adherence report scale (MARs) in Germany. J Eval Clin Pract 2010;16:574-9.

39 Löwe B, Wahl I, Rose M, et al. A 4-item measure of depression and anxiety: validation and standardization of the patient health Questionnaire-4 (PHQ-4) in the general population. J Affect Disord 2010;122:86-95.

40 Kroenke K, Spitzer RL, Williams JBW, et al. An ultra-brief screening scale for anxiety and depression: the PHQ-4. Psychosomatics 2009:50:613-21.

41 Spitzer RL, Kroenke K, Williams JBW, et al. A brief measure for assessing generalized anxiety disorder: the GAD-7. Arch Intern Med 2006;166:1092-7.

42 Kroenke K, Spitzer RL, Williams JB. The PHQ-9: validity of a brie depression severity measure. J Gen Intern Med 2001;16:606-13.

43 Dapp U, Minder CE, Anders J, et al. Long-Term prediction of changes in health status, frailty, nursing care and mortality in community-dwelling senior citizens - results from the longitudinal urban cohort ageing study (LUCAS). BMC Geriatr 2014;14:141.

44 Dapp U, Anders J, Golgert S, et al. [Resources and risks in old age: the LUCAS-I marker set for a classification of elderly people as fit, pre-frail and frail. First results on validity from the Longitudinal Urban Cohort Ageing Study (LUCAS), Hamburg]. Z Gerontol Geriatr 2012;45:262-70.

45 Lawton MP, Brody EM. Assessment of older people: selfmaintaining and instrumental activities of daily living. Gerontologist 1969:9:179-86.

46 Katz S, Ford AB, Moskowitz RW, et al. Studies of illness in the aged-The index of ADL: a standardized measure of biological and psychosocial functions. PAHO Scientific Publication 1992;534:272-80.

47 Mahoney FI, Barthel DW. Functional evaluation: the BARTHEL index. Md State Med J 1965;14:61-5.

48 Ustün TB, Chatterji S, Kostanjsek N, et al. Developing the world Health organization disability assessment schedule 2.0. Bull World Health Organ 2010;88:815-23.

49 Reitan RM. Trail making test: manual for administration and scoring. Tucson, AZ: Reitan Neuropsychology Laboratory, 1992.

50 Reitan RM. Validity of the TRAIL making test as an indicator of organic brain damage. Percept Mot Skills 1958;8:271-6.

51 Kalbe E, Kessler J, Calabrese P, et al. DemTect: a new, sensitive cognitive screening test to support the diagnosis of mild cognitive impairment and early dementia. Int $J$ Geriatr Psychiatry 2004;19:136-43.

52 Maier-Diewald W, Wittchen $\mathrm{H}-\mathrm{U}$, Hecht $\mathrm{H}$, et al. MEL - MÜNCHNER EREIGNISLISTE. München: Max-Planck-Institut für Psychiatrie, 1983.

53 Rosenbaum PR, Rubin DB. The central role of the propensity score in observational studies for causal effects. Biometrika 1983;70:41-55.

54 Mayring P. Qualitative Inhaltsanalyse. Handbuch qualitative Forschung in Der Psychologie: Reinbek bei Hamburg: Rowohlt, 2010: 601-13. 